\title{
On bifurcations of spiral waves in the plane
}

\author{
E.V.Nikolaev ${ }^{1,2}$, V.N.Biktashev ${ }^{1,2}$ and A.V.Holden ${ }^{1, *}$ \\ Submitted to IJBC 1999/01/19 \\ Accepted 1999/01/30 \\ To appear in vol. 9 no 8, Aug. 1999
}

${ }^{1}$ School of Biomedical Sciences, University of Leeds, Leeds LS2 9JT, UK

${ }^{2}$ Institute for Mathematical Problems in Biology, Pushchino, Moscow Region, 142292, Russia

* Author to whom correspondence should be addressed

\begin{abstract}
We describe the simplest bifurcations of spiral waves in reaction-diffusion systems in the plane and present the list of model systems. One-parameter bifurcations of one-armed spiral waves are fold and Hopf bifurcations. Multi-armed spiral waves may additionally undergo a period-doubling pitchfork bifurcation, when two congruent spiral wave solutions, having the 'double' period, branch from the original spiral wave at the bifurcation point.
\end{abstract}

\section{Introduction}

Rotating spiral waves occur in a variety of spatially distributed two-dimensional systems that all share the property of excitability, and have attracted attention in many fields of science since their discovery in the beginning of the nineteen-seventies [Zaikin \& Zhabotinsky 1970, Winfree 1972]. Mathematical treatment of the spiral wave phenomena is usually carried out in terms of nonlinear reaction-diffusion systems (PDEs) in two spatial dimensions. Spiral waves have wave-like and particle-like properties, their phase and a position given by the coordinates of the wavetip, and can move.

Spiral waves can rotate uniformly around fixed centers or their tips can trace out complex patterns as they rotate. Such a nonuniform rotation of the spiral wave tip has been called meander [Winfree 1973, see endnote 11]. Numerical studies have demonstrated a rich variety of meander patterns in different excitable media. Attempts to classify various types of spiral waves behaviours in homogeneous excitable media have been made by Zykov [1986] and Winfree [1991]. 
Progress in the understanding of meandering spirals was made when it was shown that transition from simple rotation to biperiodic (meandering) rotation of the wavetip corresponds to a secondary Hopf bifurcation of the spiral wave in the presence of the group of Euclidean motions in the plane (see [Barkley 1995] and earlier references therein). A classification of relative equilibria and relative periodic orbits for noncompact symmetry groups has been made by Ashwin \& Melbourne [1997]; in the context of spiral waves and Euclidean symmetry this covers uniform and biperiodic rotations.

The Hopf bifurcation of uniformly rotating spiral waves in the presence of the Euclidean group was studied by means of the Lyapunov-Schmidt reduction by Wulff [1996]. In that work a spectral assumption on the linearized problem was taken: the generalized eigenspace corresponding to critical eigenvalues was assumed to be finitedimensional. Although there is a numerical evidence that this spectral assumption may hold [Barkley 1995], a rigorous mathematical justification is still lacking.

A Hopf bifurcation of $n$-armed spiral waves, uniformly rotating waves having a cyclic spatial symmetry group of order $n$, was considered by Golubitsky, LeBlanc \& Melbourne [1997].

A center-manifold approach to the analysis of bifurcations of uniformly rotating spiral waves, including multi-armed waves, within the above-mentioned spectral assumption was outlined in [Sandstede, Scheel \& Wulff 1997].

A geometrical approach based on the separation of motions in the system by means of the group orbits reduction was used in [Biktashev, Holden \& Nikolaev 1996], where the ODE system governing the wavetip motion in the plane was obtained. The reduction has a simple 'physical' sense, as it is equivalent to passing to a moving frame of reference attached to the wavetip. In the reduced system uniform rotation of the original spiral wave (relative equilibrium) corresponds to an equilibrium and the transition to the meander (relative periodic orbit) is a standard Andronov-Hopf bifurcation of the equilibrium. This approach is not restricted to the analysis of bifurcations; so, it also allowed us to interpret the more complicated meander, which Winfree [1991] called 'hypermeander', as a consequence of a chaotic attractor in the reduced system, regardless of any scenario of its birth [Biktashev \& Holden 1998].

In the present paper, we use the center manifold approach described by Sandstede et al. [1997] and the group orbit reduction from [Biktashev et al. 1996], for a systematic study of the simplest one-parameter bifurcations of one- and multi-armed spiral waves. We also present the list of model systems capturing the most essential features of these bifurcations. Here the term 'simplest' means that the period of a spiral wave does not tend to infinity and its magnitude does not vanish at the bifurcation point.

The simplest bifurcations for one-armed spiral waves are the fold and Hopf bifurcations and multi-armed spiral waves can undergo additionally a pitchfork bifurcation. 
At the fold bifurcation point two $n$-armed spiral wave solutions merge and then disappear. The pitchfork bifurcation of an $n$-armed spiral wave is only possible for even $n$ and always results in the period-doubling for a pair of congruent spiral wave solutions appearing via the bifurcation.

The paper is organised as follows. Reaction-diffusion systems in the plane and the group of Euclidean motions of the plane are discussed in Sect. 2. Definitions of spiral waves and their symmetries are recalled in Sect. 3. Discussion of the center manifold reduction, factorization of the center manifold and separation of motions on the center manifold by symmetry, the bifurcation group, typical critical multipliers and other related topics can be found in Sec. 4. The analysis of the bifurcations and the model systems can be found in Sects. 5 and 6 .

\section{Reaction-diffusion systems in the plane}

Consider a reaction-diffusion system in the whole plane $\mathbf{R}^{2}$

$$
\partial_{t} u=\mathbf{D} \nabla^{2} u+F(u ; \alpha),
$$

where $u(\mathbf{r}, t)=\left(u_{1}(\mathbf{r}, t), \ldots, u_{l}(\mathbf{r}, t)\right), l \geq 2, \nabla^{2}$ is the Laplacian, $\mathbf{D}$ is a constant diagonal matrix of nonnegative diffusion coefficients, $F(u ; \alpha)$ is a $C^{r}$-smooth vector-function, $\alpha$ is a parameter and $\mathbf{r}=(x, y) \in \mathbf{R}^{2}$.

The fundamental property of equation (2.1) is its invariance under the group of coordinate transformations in the plane, preserving the distance between points, the Euclidean group of symmetries $\mathbf{E}(2)$. Although this group includes both motions and reflections in the plane, only the motions, i.e. rotations and translations, are important for the analysis that follows. The set of all the Euclidean motions is a subgroup of $\mathbf{E}(2)$ which we denote by $\mathbf{S E}(2)$. Given a system of Cartesian coordinates Oxy, every Euclidean motion $g \in \mathbf{S E}(2), g: \mathbf{r}=(x, y) \mapsto \tilde{\mathbf{r}}=(\tilde{x}, \tilde{y})$, can be uniquely represented by

$$
\hat{\mathbf{r}}^{\mathrm{T}}=\mathbf{L}_{\varphi} \mathbf{r}^{\mathrm{T}}+\mathbf{R}^{\mathrm{T}}, \quad \mathbf{L}_{\varphi}=\left(\begin{array}{rr}
\cos \varphi & -\sin \varphi \\
\sin \varphi & \cos \varphi
\end{array}\right), \quad \mathbf{R}=\left(R_{1}, R_{2}\right) .
$$

Here $\mathbf{L}_{\varphi}$ is rotation through angle $\varphi \in \mathbf{R} / 2 \pi \mathbf{Z}$ around the origin of the coordinate system $O x y$, followed by parallel translation $\mathbf{R}$ along vector $\left(R_{1}, R_{2}\right)$. Recall that the group $\mathbf{S E}(2)$ is a semidirect product of its two subgroups, $\mathbf{S E}(2)=\mathbf{T}(2) \star \mathbf{S O}(2)$, where $\mathbf{T}(2) \cong \mathbf{R}^{2}$ is the subgroup of translations in the plane and $\mathbf{S O}(2)$ is the subgroup of rotations of the plane. Each $g \in \mathbf{S E}(2)$ can be uniquely defined by the three parameters $R_{1}, R_{2}$ and $\varphi$ that we view as coordinates on $\mathbf{S E}(2), \operatorname{dim} \mathbf{S E}(2)=3$. 
Let $\mathcal{B}$ be a functional space and let us define the action $T$ of $\mathbf{S E}(2)$ on $\mathcal{B}$ by

$$
T(g) u(\mathbf{r})=u\left(g^{-1} \mathbf{r}\right), \quad u(\mathbf{r}) \in \mathcal{B} .
$$

Then all linear transformations $T(g), g \in S E(2)$, of the space $B$, constitute the symmetry group of the differential equation (2.1): any $T(g)$ transforms any solution to (2.1) to a solution.

To study solutions of (2.1), an appropriate functional space $\mathcal{B}$ is to be defined. In general, the spiral waves do not vanish at infinity, so the functional space $L_{2}\left(\mathbf{R}^{2}, \mathbf{R}^{l}\right)$ does not fit the case. We suppose that (2.1) is posed on the space of bounded uniformly continuous vector-functions $\mathcal{B}=B C_{\text {unif }}\left(\mathbf{R}^{2}, \mathbf{R}^{l}\right)$. Then solutions to the equation (2.1) are $C^{r}$-smooth in time $t>0$, space $\mathbf{r}$, initial conditions, and the parameter $\alpha$ [Henry 1981].

\section{Spiral waves}

\subsection{One- and multi-armed spiral waves}

Consider a $\tau$-periodic solution $u(\mathbf{r}, t)$ to $(2.1)$, representing a wave uniformly rotating around the origin $O, \mathbf{r}_{0}=(0,0)$. Throughout this section, we omit the explicit dependence of both equation (2.1) and its solutions upon the parameter $\alpha$ in the notation. Let $u(\mathbf{r}, t)$ have spatial symmetry $K$ isomorphic to $\mathbf{Z}_{n}$ and let, be the generator of $K=\left\{,{ }^{j}, j=0, \ldots, n-1\right\}$, corresponding to rotation of the plane through angle $\varphi_{n}=2 \pi / n$. By definition, $u(\mathbf{r}, t) \equiv u(\mathbf{r}, t)$. In polar coordinates $(\rho, \theta), x=\rho \cos \theta, y=\rho \sin \theta$, the uniform rotation is described by

$$
u(\rho, \theta, t)=U(\rho, \theta-\omega t)
$$

where $\omega$ is the angular velocity and $U(\rho, \tilde{\theta})$ is periodic in $\tilde{\theta}=\theta-\omega t$. Using (2.2) we get

$$
u(\rho, \theta, t) \equiv u\left(\rho, \theta-\varphi_{n}, t\right) \equiv u\left(\rho, \theta, t+\frac{2 \pi}{n \omega}\right) \equiv u(\rho, \theta, t)
$$

(for simplicity throughout this paper we always use the same notation for representations of transformation, in different coordinate systems). So, $u(\mathbf{r}, t)$ and $U(\rho, \tilde{\theta})$ are periodic in their second arguments with periods $\tau=2 \pi /(n \omega)$ and $2 \pi / n$ respectively.

Definition. A periodic solution of the form (3.1) with the period $\tau=2 \pi /(n \omega)$ and the cyclic spatial symmetry group $K$ of order $n$ is called an $n$-armed spiral wave uniformly rotating with frequency $\omega$ around the symmetry center $\mathbf{r}_{0}=(0,0)$ in the plane. 
Thus, every arm of the spiral wave uniformly rotates with the frequency $\omega$ and the period $n \tau$, where $\tau$ is the period of the corresponding spiral wave solution $u(\mathbf{r}, t)$.

Remark. The definition given above does not always agree with the usual meaning of the term ' $n$-armed spiral wave'. So, we also use the term ' $\mathbf{Z}_{d}$-symmetric spiral wave' for an $n$-armed spiral wave with the spatial symmetry of order $d$, a divisor of $n$. For instance, a slight perturbation of an $n$-armed spiral will not, in the usual sense, change the number of its arms, but may break its spatial symmetry.

\subsection{Spiral waves in rotating frames of reference}

For an $n$-armed spiral wave $u(\mathbf{r}, t)$ with the period $\tau=2 \pi /(n \omega)$ and the spatial symmetry $K$ to exist, there should exist a stationary solution $U(\tilde{\mathbf{r}}), U(\tilde{\mathbf{r}})=U(\tilde{\mathbf{r}})$, in the modified system

$$
\partial_{t} v=\mathbf{D} \tilde{\nabla}^{2} v+F(v)+\omega \partial_{\tilde{\theta}} v
$$

The system (3.2) is the original system (2.1) rewritten in a frame of reference that is rotating with the frequency $\omega$. Here $\tilde{\mathbf{r}}=(\hat{x}, \hat{y}), \tilde{\nabla}^{2}$ is the Laplacian and $\partial_{\tilde{\theta}}=\tilde{x} \partial_{\tilde{y}}-\tilde{y} \partial_{\tilde{x}}$ is the operator of differentiation by the angular variable in the rotating frame of reference.

Let $A_{1}=-\partial_{\tilde{x}}, A_{2}=-\partial_{\tilde{y}}$ and $A_{3}=-\partial_{\tilde{\theta}}$ be the infinitesimal generators of the action (2.2) in the rotating frame of reference as above. Consider the eigenvalue problem on complex space $\mathcal{B} \oplus i \mathcal{B}$ :

$$
L V(\tilde{\mathbf{r}}) \equiv\left(\mathbf{D} \tilde{\nabla}^{2}+\partial_{v} F(U(\hat{\mathbf{r}}))-\omega A_{3}\right) V(\tilde{\mathbf{r}})=\lambda V(\tilde{\mathbf{r}})
$$

Since $T(g) F(v)=F(T(g) v)$, we get $A_{i} F=\partial_{v} F A_{i}(j=1,2,3)$. Using $\mathbf{D} \tilde{\nabla}^{2} U(\tilde{\mathbf{r}})+$ $F(U(\hat{\mathbf{r}}))=\omega A_{3} U(\hat{\mathbf{r}})$, one can obtain

$$
L A_{i} U(\tilde{\mathbf{r}})=\omega\left[A_{i}, A_{3}\right] U(\tilde{\mathbf{r}}),
$$

where $\left[A_{i}, A_{3}\right]=A_{i} A_{3}-A_{3} A_{i}$. It can be easily checked that $L$ always has the three eigenvalues 0 and $\pm i \omega$. These eigenvalues correspond to the rotation eigenmode $\eta_{0}(\tilde{\mathbf{r}})=$ $\partial_{\tilde{\theta}} U(\tilde{\mathbf{r}})$ and the translation eigenmodes $\eta_{ \pm}(\tilde{\mathbf{r}})=\left(\partial_{\tilde{x}} \pm i \partial_{\tilde{y}}\right) U(\tilde{\mathbf{r}})$, respectively.

Recall that $\mu=\mathrm{e}^{\lambda \tau}$ and $\lambda$ are called the multipliers and the Floquet exponents of the periodic orbit $u(\mathbf{r}, t)$, respectively. Thus, any $n$-armed spiral wave has always the three trivial multipliers, 1 and $\mathrm{e}^{ \pm 2 \pi i / n}$, lying on the unit circle in the complex plane. 


\subsection{Group manifolds of spiral waves}

Let $u(\mathbf{r}, t)$ be a spiral wave uniformly rotating around $\mathbf{r}_{0}=\left(x_{0}, y_{0}\right)$ in the plane. Then $\tilde{u}(\mathbf{r}, t)=T(g) u(\mathbf{r}, t)$ is also a spiral wave, that rotates around $\tilde{\mathbf{r}}_{0}^{\mathrm{T}}=\mathbf{L}_{-\varphi}\left(\mathbf{r}_{0}^{\mathrm{T}}-\mathbf{R}^{\mathrm{T}}\right)$. So, spiral waves $T(g) u(\mathbf{r}, t), g \in \mathbf{S E}(2)$, form a manifold $\mathcal{S}, \operatorname{dim} \mathcal{S}=\operatorname{dim} \mathbf{S E}(2)=$ 3 , which can be parametrised by the coordinates $\left(R_{1}, R_{2}, \varphi\right)$, representing different rotation centers $\mathbf{r}_{0}$ and rotation phases $\varphi$. In particular, it follows that the three eigenvalues 0 and $\pm i \omega$ of the eigenvalue problem (3.3) characterise the neutral stability of the spiral waves with respect to shifts along $\mathcal{S}$.

Since solutions to the equation (2.1) are $C^{r}$-smooth in space $\mathbf{r}$, see Sect. 2, translations in the plane act $C^{r}$-smoothly on the solutions. By definition, time evolution of a uniformly rotating spiral wave coincides with its rotation. Recall that solutions to (2.1) are $C^{r}$-smooth in time $t>0$. It follows that rotation of the plane acts $C^{r}$-smoothly on the spiral wave. Thus, the manifold of spiral waves $\mathcal{S}$, generated by the translations and rotations of a given spiral wave solution, is $C^{r}$-smooth.

\subsection{Symmetry in families of spiral waves}

Let $u(\mathbf{r}, t ; \alpha)$ be a family of spiral waves smoothly depending on the parameter $\alpha$. Then the finite spatial symmetry group $K(\alpha)$ of $u(\mathbf{r}, t ; \alpha)$ does not depend on $\alpha$ until $u\left(\mathbf{r}, t ; \alpha_{0}\right)$ has a non-trivial unit multiplier at some branching point $\alpha=\alpha_{0}$ [Nikolaev $\&$ Shnol 1998a]. At branching points several families of spiral waves with the same or different symmetry groups $\tilde{K}(\alpha)$ may merge and so, their symmetry groups may increase at $\alpha_{0}$ : all groups $\tilde{K}(\alpha)$ are subgroups of the symmetry group $K_{0}$ of the critical spiral wave at $\alpha_{0}$. This does not happen in generic one-parameter families of periodic solutions with finite symmetry groups: there always exists a family of periodic orbits that have exactly the maximal spatial symmetry $K_{0}$ for all $\alpha$ near $\alpha_{0}$, as long as the periodic orbits exist [Nikolaev \& Shnol 1998b]. In this paper we always suppose that the given family $u(\mathbf{r}, t ; \alpha)$ is such a maximally symmetric family of spiral wave periodic solutions. We note that for the bifurcation analysis of the spiral wave periodic solution $u\left(\mathbf{r}, t ; \alpha_{0}\right)$, only its spatial symmetry group $K_{0}$ at the bifurcation point $\alpha_{0}$ is important.

\section{The center manifold reduction}

\subsection{On the center manifold and the reduced system}

Let a spiral wave periodic solution $u(\mathbf{r}, t ; 0)$ with the spatial symmetry $K$ of order $n$ have a finite number $s_{0} \geq 1$ of isolated non-trivial multipliers $\mu=\mathrm{e}^{\lambda \tau}$ with $\operatorname{Re} \lambda=0$ at $\varepsilon=0$. Here $\varepsilon=\alpha-\alpha_{0}$ is a new parameter and $\alpha_{0}$ is a critical value of the original 
parameter $\alpha$. Let these multipliers correspond to eigenfunctions $V_{0}(\tilde{\mathbf{r}})$ satisfying (3.3) at $\varepsilon=0$. We call $V_{0}(\tilde{\mathbf{r}})$ a critical eigenmode, $\lambda$ a critical eigenvalue and $\mu$ a critical multiplier. Then there exists an SE(2)-invariant $C^{r-1}$-smooth center manifold $\mathcal{M}_{c}(\varepsilon)$ [Sandstede et al. 1997, Theorem 4]. For definition of the center manifold depending on the parameter see, for instance, $\$ 5.2$ in [Kuznetsov 1995]. We denote by $C_{\varepsilon}$ the periodic orbit corresponding to the spiral wave, $C_{\varepsilon} \subset \mathcal{M}_{c}(\varepsilon)$, and by $v_{0}(\varepsilon)$ a point of $C_{\varepsilon}$.

Due to the slice theorem [Mielke 1991, Theorem 5.1], which we formulate below as Lemmas 4.2.1 and 4.3.1, there exists a $C^{k-1}$-smooth, $s_{0}$-dimensional submanifold $M_{s}(\varepsilon)$ of $\mathcal{M}_{c}(\varepsilon)$ such that

(i) $M_{s}(\varepsilon)$ is $K$-invariant and is transversal to group orbits starting within $M_{s}(\varepsilon)$;

(ii) $\mathcal{M}_{c}(\varepsilon)$ is foliated by transporting $M_{s}(\varepsilon)$ under the action of $\mathbf{S E}(2)$;

(iii) in the case of one-armed spiral waves $\mathcal{M}_{c}(\varepsilon)$ is diffeomorphic to the direct product $\mathbf{S E}(2) \times M_{s}(\varepsilon)$ and in the case of $n$-armed spiral waves $\mathbf{S E}(2) \times M_{s}(\varepsilon)$ is an $n$ sheeted covering of $\mathcal{M}_{c}(\varepsilon)$.

The induced action of the group $\mathbf{S E}(2)$ on $\mathbf{S E}(2) \times M_{s}(\varepsilon)$ corresponds to left shifts of $\mathbf{S E}(2)$ on the first component and is identical on the second component of the direct product.

The system induced on the direct product $\mathbf{S E}(2) \times M_{s}(\varepsilon)$ takes a simple (skew product) form, see below. This allows one to reduce the bifurcation analysis of the original $\left(s_{0}+3\right)$-dimensional system on the center manifold $\mathcal{M}_{c}(\varepsilon)$ to the bifurcation analysis of an appropriate equilibrium of the corresponding $s_{0}$-dimensional subsystem on the submanifold $M_{s}(\varepsilon)$.

Remark. The results formulated in Sects. 4.2 and 4.3 are not new and can be found in [Mielke 1991, Biktashev et al. 1996, Fiedler, Sandstede, Scheel \& Wulff 1996, Sandstede et al. 1997]. We formulate and prove them just for completeness and convenience of the reader.

\subsection{The system on the center manifold: the case of one-armed spiral waves}

\subsubsection{Factorization of the center manifold by symmetry}

Lemma 4.2.1 1. There exists a $C^{k-1}$-smooth submanifold $M_{s}(\varepsilon)$ of the center manifold $\mathcal{M}_{c}(\varepsilon), \operatorname{dim} M_{s}(\varepsilon)=s_{0}$, transversal to group orbits starting within $M_{s}(\varepsilon)$. 
2. There exists a diffeomorphism $\theta: \mathcal{M}_{c}(\varepsilon) \rightarrow \mathbf{S E}(2) \times M_{s}(\varepsilon)$ such that the induced action $S(\tilde{g})$ of $\mathbf{S E}(2)$ on the direct product $\mathbf{S E}(2) \times \mathcal{M}_{c}(\varepsilon)$ is given by

$$
\begin{cases}\theta \circ T(\tilde{g})=S(\tilde{g}) \circ \theta, & \theta(w)=(g, v), \\ S(\tilde{g})(g, v)=(\tilde{g} g, v), & \tilde{g}, g \in \mathbf{S E}(2) .\end{cases}
$$

Here $w$ is a coordinate on $\mathcal{M}_{c}(\varepsilon), w \in W, W$ is an unbounded domain of $\mathbf{R}^{s_{0}+3}$, $\operatorname{dim} W=s_{0}+3 ; v$ is a coordinate on $M_{s}(\varepsilon), v \in V$, and $V$ is a bounded subdomain of $W, \operatorname{dim} V=s_{0}$.

\section{Remarks.}

- The submanifold $M_{s}(\varepsilon)$ is called the quotient manifold of the center manifold $\mathcal{M}_{c}(\varepsilon)$ with respect to the symmetry group $\mathbf{S E}(2), M_{s}(\varepsilon)=\mathcal{M}_{c}(\varepsilon) / \mathbf{S E}(2)$.

- The submanifold $M_{s}(\varepsilon)$ is also called a slice and Lemma 4.2.1 is a particular case of a general slice theorem, see Theorem 5.1 in [Mielke 1991] and references therein. We have formulated and will prove the lemma just for completeness since both lemma and its proof will be used in what follows.

Proof of the Lemma. Denote by $X$ the tangent space to $\mathcal{M}_{c}(\varepsilon)$ at the point $v_{0}(\varepsilon)$, $X$ is isomorphic to $\mathbf{R}^{s_{0}+3}$. We keep the same notation $T(g)$ for the action $T(g)$ of $\mathbf{S E}(2)$ restricted to $X$. Let $Y$ be the tangent space to the group orbits manifold $\mathcal{S}$ at the point $v_{0}(\varepsilon), \mathcal{S}=\left\{w=T(g) v_{0}(\varepsilon): g \in \mathbf{S E}(2)\right\}$. Then $X$ may be decomposed into the direct $\operatorname{sum} X=Y \oplus Z$. Denote by $\psi_{\varepsilon}$ a $C^{k-1}$-smooth chart, a local $C^{k-1}$-smooth diffeomorphism of a small neighbourhood of $v_{0}(\varepsilon)$ onto a small domain of $X$. We keep the same notation $v_{0}(\varepsilon)$ for the image $\psi_{\varepsilon}\left(v_{0}(\varepsilon)\right) \in X$ of the point $v_{0}(\varepsilon) \in \mathcal{M}_{c}(\varepsilon)$ and identify the origin of $X$ with $v_{0}(\varepsilon)$. Since $T(g) v_{0}(\varepsilon) \neq v_{0}(\varepsilon)$ for all $g \in \mathbf{S E}(2)$, $g \neq e$, where $e$ is the unit element of $\mathbf{S E}(2)$, and $\|T(g) v(\varepsilon)-v(\varepsilon)\| \rightarrow 0$ as $g \rightarrow e$ it follows that for any sufficiently small $\delta>0$ there exist small neighbourhoods $U_{\delta}$ and $U_{2 \delta}$ of $e, U_{\delta} \subset U_{2 \delta} \subset \mathbf{S E}(2)$, such that $\|T(g) v(\varepsilon)-v(\varepsilon)\| \geq \delta$ for all $g \notin U_{\delta}$ and $\|T(g) v(\varepsilon)-v(\varepsilon)\| \geq 2 \delta$ for all $g \notin U_{2 \delta}$. Let $\tilde{V}$ be a small neighbourhood of $v_{0}(\varepsilon)$ within $Z, \tilde{V} \subset Z$. Due to the decomposition $X=Y \oplus Z$ the domain $\tilde{V}$ is transversal to group orbits starting within $\tilde{V}$. It follows that $\delta$ and $\tilde{V}$ may be chosen so small that $T(g) \tilde{V} \cap \tilde{V}=\emptyset$ for all $g \in U_{2 \delta}$ and $g \neq e$. Let $B_{0}, B_{0} \subset X$, be a small ball with the radius $r_{0}<\delta / 2$, centered at $v_{0}(\varepsilon)$. Then $T(g) B_{0} \cap B_{0}=\emptyset$ for all $g \notin U_{\delta}$. Let us choose a subdomain $V \subset B_{0} \cap \tilde{V}$ then $T(g) V \cap V=\emptyset$ for all $g \notin U_{\delta}$ or $g \in U_{2 \delta}, g \neq e$, and, hence, $T(g) V \cap V=\emptyset$ for all $g \in \mathbf{S E}(2)$ and $g \neq e$. 
The needed submanifold $M_{s}(\varepsilon)$ is then the inverse image of $V$ under the coordinate diffeomorphism $\psi_{\varepsilon}$, i.e. $M_{s}(\varepsilon)=\left\{\psi_{\varepsilon}^{-1}(v) \in \mathcal{M}_{c}(\varepsilon): v \in V\right\}$. Due to the choice of $V$, every $w \in W, W=\{T(g) V: g \in \mathbf{S E}(2)\}$, may be uniquely represented in the form $w=T(g) v$. The points $w$ of $W$ may be viewed as global coordinates on the center manifold $\mathcal{M}_{c}(\varepsilon)$. We define $\theta(w)=(g, v)$ by $\theta^{-1}(g, v)=T(g) v$. The formulas (4.1) follow from $T(\tilde{g}) w=T(\tilde{g} g) v$. The proof follows.

\subsubsection{Decomposition of motion on the center manifold}

Due to the invariance of the system reduced to the center manifold $\mathcal{M}_{c}(\varepsilon)$ with respect to the action of the group $\mathbf{S E}(2)$, the motion on the direct product $\mathbf{S E}(2) \times V$ may be decomposed into superposition of motions along $\mathbf{S E}(2)$ and along $V$ as follows.

Lemma 4.2.2 The system on the center manifold $\mathcal{M}_{c}(\varepsilon)$, rewritten in the coordinates $(g, v)=\theta(w)$, has the following form.

$$
\left\{\begin{array}{l}
(a) \quad \dot{\varphi}=w(v ; \varepsilon), \\
(b) \quad \dot{R}=\mathrm{e}^{i \varphi} a(v ; \varepsilon), \\
(c) \quad \dot{v}=b(v ; \varepsilon) .
\end{array}\right.
$$

Here $R=\hat{R}_{1}+i \hat{R}_{2},\left(\varphi, \hat{R}_{1}, \hat{R}_{2}\right)$ are coordinates on $\mathbf{S E}(2), g=\left(\varphi, \hat{R}_{1}, \hat{R}_{2}\right), \varphi \in \mathbf{R} / 2 \pi \mathbf{Z}$, $\left(\hat{R}_{1}, \hat{R}_{2}\right) \in \mathbf{R}^{2}$, and $v$ is a real coordinate on $V ; a(v ; \varepsilon)=a_{1}(v ; \varepsilon)+i a_{2}(v ; \varepsilon)$.

\section{Remarks.}

- The subsystem $(4.2(c))$ is called a quotient system and may be directly obtained from the original system by factoring out the symmetry group $\mathbf{S E}(2)$, for more details see [Biktashev et al. 1996]. Thus, the change of coordinates $(g, v)=\theta(w)$ corresponds to the factorization of the original system by the symmetry.

- Generically, the quotient system has no additional symmetry group.

Before giving the proof of Lemma 4.2.2, we recall some definitions and known facts. Given a manifold $M$ and a point $m$ of $M$, we denote by $T_{m} M$ the tangent space to $M$ at $m$. A Lie group $G$ is a smooth manifold with $G$ smoothly acting on itself by left shifts. In this representation, a group element $g$ maps the neighbourhood of the unit element $e \in G$ onto the neighbourhood of $g$. The linearisation of this map is an isomorphism of the correspondent tangent spaces $T_{e} G$ and $T_{g} G$, which is called a tangent map and is denoted by $g_{*}, g_{*}: T_{e} G \rightarrow T_{g} G$. A vector field $\mathcal{F}$ on the phase space $X$ is invariant 
under the linear action $T(g)$ of $G$ on $X$ if the identity $\mathcal{F}(T(g) x)=T(g) \mathcal{F}(x)$ holds for all $g \in G$ and $x \in X$. A vector field $f$ on a Lie group $G$ is invariant under left shifts on $G$ if $f(g h)=g_{*} f(h)$ for all $g, h \in G$. Any such vector field is uniquely defined by the vector $f(e)$ at the unit element $e$ of $G: f(g)=g_{*} f(e)$.

Proof of the lemma. Since the original vector field on $\mathcal{M}_{c}(\varepsilon)$ in w-coordinates is ' $T(g)$-invariant' and the change of coordinates $(g, v)=\theta(w)$ commutes with the action of the symmetry group $\mathbf{S E}(2)$ as given in (4.1), the vector field on $\mathcal{M}_{c}(\varepsilon)$, rewritten in $(g, v)$-coordinates, is ' $S(g)$-invariant'. Recall that the group $\mathbf{S E}(2)$ acts identically on $V$, see (4.1). It follows that the $V$-component of the vector field on $\mathbf{S E}(2) \times V$ does not depend on g, i.e. (4.2(c)) holds. Due to (4.1) the $\mathbf{S E}(2)$-component of the vector field on $\mathbf{S E}(2) \times V$ is invariant under left shifts of $\mathbf{S E}(2)$ on itself and, hence, may be uniquely extended over the whole manifold $\mathbf{S E}(2)$ by the tangent map $g_{*}$, see above.

To obtain equations $(4.2(\mathrm{a}),(\mathrm{b}))$ let us write down explicitly a left shift $g=(\varphi, R)$ on $\mathbf{S E}(2)$ :

$$
g:\left(\varphi_{1}, R_{1}\right) \mapsto\left(\varphi_{2}, R_{2}\right), \quad \varphi_{2}=\varphi_{1}+\varphi, R_{2}=R_{1} \mathrm{e}^{i \varphi}+R .
$$

The tangent map $g_{*}: T_{g_{1}} \mathbf{S E}(2) \rightarrow T_{g_{2}} \mathbf{S E}(2)$, in the complex bases $\partial_{\varphi_{j}}, \partial_{R_{j}}, j=1,2$, is the Jacobi matrix of the transformation (4.3),

$$
g_{*}=\left(\begin{array}{cc}
1 & 0 \\
0 & \mathrm{e}^{i \varphi}
\end{array}\right) \text {. }
$$

Let $(\omega(v ; \varepsilon), a(v ; \varepsilon), b(v ; \varepsilon))$ be the coordinates of the vector at point $(e, v)$ of $\mathbf{S E}(2) \times V$, where $(\omega(v ; \varepsilon), a(v ; \varepsilon))$ are the corresponding coordinates of the $\mathbf{S E}(2)$-component of this vector in the basis $\partial_{\varphi}, \partial_{R}$ of $T_{e} \mathbf{S E}(2)$. Then using (4.4), we obtain

$$
g_{*}:(\omega(v), a(v)) \mapsto\left(\omega(v), \mathrm{e}^{i \varphi} a(v)\right),
$$

which gives $(4.2(\mathrm{a}),(\mathrm{b}))$. The proof follows.

\subsection{The system on the center manifold: the case of multi- armed spiral waves}

\subsubsection{On the impossibility of factorization of the center manifold by sym- metry}

In this case the original $n$-armed spiral wave periodic orbit $C_{\varepsilon}$ has the spatial symmetry group $K$ of order $n, n>1$. An important consequence of this fact is that Lemmas 4.2.1 and 4.2 .2 cannot be applied to the case directly. Indeed, the lemmas result from 
factorization of both center manifold $\mathcal{M}_{c}(\varepsilon)$ and the system on $\mathcal{M}_{c}(\varepsilon)$ by symmetry. To do this the symmetry group $\mathbf{S E}(2)$ should act freely on $\mathcal{M}_{c}(\varepsilon)$, that is $T(g) w \neq w$ for all $g \in \mathbf{S E}(2), g \neq e$, and $w \in W$, see Lemma 4.2.1. Since, $v=v$ for all $v \in C_{\varepsilon}$, ,$\in K$, the group $\mathbf{S E}(2)$ does not act freely on $\mathcal{M}_{c}(\varepsilon)$ and, hence, the latter cannot be factorized by the action of $\mathbf{S E}(2)$. Here, is the generator of the cyclic group $K$, corresponding to rotation of the plane through angle $2 \pi / n$.

Below we show that in this case there also exits a submanifold $M_{s}(\varepsilon)$ of $\mathcal{M}_{c}(\varepsilon)$ such that $M_{s}(\varepsilon)$ is invariant under $K, M_{s}(\varepsilon)=M_{s}(\varepsilon)$, and is transversal to group orbits of $\mathbf{S E}(2)$ starting within $M_{s}(\varepsilon)$. As in Lemma 4.2.1, we identify $\mathcal{M}_{c}(\varepsilon)$ with the (unbounded) domain $W$ and $M_{s}(\varepsilon)$ with the (bounded) domain $V, V \subset W$.

It has been noticed in [Fiedler et al. 1996] that the direct product $\mathbf{S E}(2) \times V$ is an $n$-sheeted covering of $W$ with the covering map $\Pi, \Pi: \mathbf{S E}(2) \times V \rightarrow W$, given by $w=\Pi(g, v)=T(g) v$. By definition of an $n$-sheeted covering, see Chapt. 4, $\S 18$ in [Dubrovin, Fomenko \& Novikov 1984], the covering space $\mathbf{S E}(2) \times V$ consists of $n$ congruent copies (sheets) of the base space $W$. The covering map $\Pi$ restricted to a sheet is a diffeomorphism. We denote by $\Pi_{*}$ the corresponding tangent map. Then the given vector field on $W$ may be lifted in a standard way to an $\mathbf{S E}(2)$-invariant vector field on the covering space $\mathbf{S E}(2) \times V$ by the map $\Pi_{*}^{-1}$.

Since the symmetry group $\mathbf{S E}(2)$ acts freely on the $n$-sheeted covering $\mathbf{S E}(2) \times V$ of the center manifold $\mathcal{M}_{c}(\varepsilon)$, Lemmas 4.2.1 and 4.2.2 may be now applied directly to both the chosen covering manifold and the system lifted to the covering manifold, see Secs. 4.3.2-4.3.4. Knowing the dynamics of the system on $\mathbf{S E}(2) \times V$, it is easy to find the dynamics on the base space $W$ : the covering map $\Pi$ sends a trajectory $(g(t), v(t))$ on $\mathbf{S E}(2) \times V$ to the corresponding trajectory $w(t)=\Pi(g(t), v(t))=T(g(t)) v(t)$ on the base space $W$.

\subsubsection{Auxiliary geometric constructions}

Lemma 4.3.1 There exists a $C^{k-1}$-smooth submanifold $M_{s}(\varepsilon)$ of the center manifold $\mathcal{M}_{c}(\varepsilon)$ such that

1. $M_{s}(\varepsilon)$ is transversal to the group orbits starting within $M_{s}(\varepsilon)$.

2. $M_{s}(\varepsilon)$ is $K$-invariant.

3. $K$ is the maximal symmetry of $M_{s}(\varepsilon)$, i.e. $T(g) M_{s}(\varepsilon) \cap M_{s}(\varepsilon)=\emptyset$ for all $g \notin K$.

Proof. This lemma is proved similarly to Lemma 4.2.1. We use the same notations as in the proof of Lemma 4.2.1. 
1. Let $Y$ be the tangent space to the group orbits manifold $\mathcal{S}$ at the point $v_{0}(\varepsilon)$. Since both $v_{0}(\varepsilon)$ and $\mathcal{S}$ are $K$-invariant, $Y$ is also $K$-invariant. Let $\left\langle w_{1}, w_{2}\right\rangle$ be an arbitrary scalar product on the tangent space $X$ to $\mathcal{M}_{c}(\varepsilon)$ at $v_{0}(\varepsilon), X$ is $K$-invariant. Then the scalar product $\left(w_{1}, w_{2}\right)$ defined as $\left.\left(w_{1}, w_{2}\right)=\sum_{g \in K}<T(g) w_{1}, T(g) w_{2}\right\rangle$, is $K$-invariant, i.e. $\left(T(g) w_{1}, T(g) w_{2}\right)=\left(w_{1}, w_{2}\right)$ for all $g \in K$ and $w_{j} \in X$. Using the $K$-invariant scalar product, one may decompose $X$ into the orthogonal sum of two $K$-invariant subspaces, $X=Y \oplus Z$. Let $\tilde{B}_{0}, \tilde{B}_{0} \subset X$, be a small neighbourhood of $v_{0}(\varepsilon)$. Then $B_{0}=\bigcap_{g \in K} T(g) \tilde{B}_{0}$ is $K$-invariant. Let $V=B_{0} \cap Z$ then the needed submanifold $M_{s}(\varepsilon)$ is the inverse image of $V$ under the coordinate diffeomorphism $\psi_{\varepsilon}$, $M_{s}(\varepsilon)=\left\{\psi_{\varepsilon}^{-1}(v) \in \mathcal{M}_{c}(\varepsilon): v \in V\right\}$. The transversality of $M_{s}(\varepsilon)$ to group orbits starting within $M_{s}(\varepsilon)$ follows from the decomposition $X=Y \oplus Z$.

2. Since $K$ is a finite (compact) group and both $v_{0}(\varepsilon)$ and $V$ are $K$-invariant, the diffeomorphism $\psi_{\varepsilon}$ may be chosen to commute with transformations of $K$ [Ruelle 1973], i.e. the chosen submanifold $M_{s}(\varepsilon)$ is $K$-invariant.

3. Since $T(g) v_{0}(\varepsilon) \neq v_{0}(\varepsilon)$ for all $g \notin K$, for any sufficiently small $\delta>0$ there exist small neighbourhoods $U_{k, \delta}$ and $U_{k, 2 \delta}$ of each $k \in K$ such that $U_{k, \delta} \subset U_{k, 2 \delta}$ and the inequalities $\left\|T(g) v_{0}(\varepsilon)-v_{0}(\varepsilon)\right\|>\delta$ for all $g \notin U_{\delta}$ and $\left\|T(g) v_{0}(\varepsilon)-v_{0}(\varepsilon)\right\|>2 \delta$ for all $g \notin U_{2 \delta}$ hold. Here $U_{\delta}=\bigcup_{k \in K} U_{k, \delta}$ and $U_{2 \delta}=\bigcup_{k \in K} U_{k, 2 \delta}, U_{k}=k U_{e}$. Let $\tilde{V}$ be a small neighbourhood of $v_{0}(\varepsilon)$ within $Z$. Due to transversality of $\tilde{V}$ to group orbits starting within $\tilde{V}$, both $\tilde{V}$ and $\delta>0$ may be chosen so small that $T(g) \tilde{V} \cap \tilde{V}=\emptyset$ for all $g \in U_{2 \delta}$ and $g \notin K$. Let now $B_{0}$ be a ball with the radius $r_{0}<\delta / 2$, centered at $v_{0}(\varepsilon)$. Then $T(g) B_{0} \cap B_{0}=\emptyset$ for all $g \notin U_{\delta}$. Let $V \subset B_{0} \cap \tilde{V}$ then $T(g) V \cap V=\emptyset$ for all $g \in \mathbf{S E}(2)$ and $g \notin K$. Thus, $T(g) V=V$ only for all $g \in K$, i.e. $K$ is the maximal symmetry of $V$. The proof follows.

Lemma 4.3.2 1. The direct product $\mathbf{S E}(2) \times V$ is an n-sheeted covering of the center manifold $\mathcal{M}_{c}(\varepsilon)$ with the covering map $\Pi$ given by

$$
w=\Pi(g, v)=T(g) v
$$

where $w$ is a coordinate on $\mathcal{M}_{c}(\varepsilon), w \in W$, and $(g, v) \in \mathbf{S E}(2) \times V$.

2. The covering map $\Pi$ induces the action $S(\tilde{g})$ of $\mathbf{S E}(2)$ on $\mathbf{S E}(2) \times V, S(\tilde{g})(g, v)=$ $(\tilde{g} g, v)$, given by the commutation relationship

$$
T(\tilde{g}) \circ \Pi=\Pi \circ S(\tilde{g}), \quad \tilde{g} \in \mathbf{S E}(2) .
$$


Proof. 1. For each pair $(g, v) \in \mathbf{S E}(2) \times V$ consider the set $S_{K}(g, v)$ of $n$ points given by $S_{K}(g, v)=\left\{\left(g k^{-1}, T(k) v\right): k \in K\right\}$. Let us identify all points of the set $S_{K}(g, v)$ with the point $w=T(g) v, w \in W$. There is a one-to-one correspondence between points $w$ of $W$ and $K$-orbits $S_{K}(g, v)$. Indeed, by definition of $W$ (see the proof of Lemma 4.2.1), for any $w \in W$ there exists a pair $(g, v) \in \mathbf{S E}(2) \times V$ such that $w=T(g) v$. It follows that $\Pi$ sends $\mathbf{S E}(2) \times V$ onto $W$. Let $w=T\left(g_{1}\right) v_{1}$ for some $\left(g_{1}, v_{1}\right) \in \mathbf{S E}(2) \times V$, where $w=T(g) v$. Then we have $v_{1}=T\left(g_{1}^{-1} g\right) v$. Since $K$ is the maximal symmetry of $V$, see Lemma 4.3.1, we obtain $g_{1}^{-1} g=k \in K$, that is $g_{1}=g k^{-1}$, $v_{1}=T(k) v$ and, therefore, $\left(g_{1}, v_{1}\right) \in S_{K}(g, v)$. Thus, by definition, the direct product $\mathbf{S E}(2) \times V$ is an $n$-sheeted covering of $W$ with the covering map $\Pi$ given by (4.6). Indeed, $\Pi\left(g k^{-1}, T(k) v\right)=T\left(g k^{-1}\right) T(k) v=T(g) v$ and therefore, $\Pi: S_{K}(g, v) \rightarrow w$.

2. The commutation relationship (4.7) follows from (4.6). The proof follows.

Remark. Notice that the sets $S_{K}(g, v)$ are orbits of the group $\tilde{K}$ of order $n$ with the action on $\mathbf{S E}(2) \times V$ given by $\tilde{k}(k, g, v)=\left(g k^{-1}, T(k) v\right)$, where $k \in K$, see the proof of Lemma 4.3.2. The group $\tilde{K}$ permutes the sheets of the covering $\mathbf{S E}(2) \times V$ among each other. Thus, the covering space $\mathbf{S E}(2) \times V$ is invariant under the group $\mathbf{S E}(2) \times \tilde{K}$ with the action of these two groups as described above. The triple $(W, \mathbf{S E}(2) \times V, \Pi)$ with the covering map $\Pi$ determined by a freely acting discrete group $\tilde{K}$ is called a principal fibre bundle with a structural group $\tilde{K}$, see Chapt. 6, $\S 24$ in [Dubrovin et al. 1984].

\subsubsection{The system on $\mathbf{S E}(2) \times V$}

Let $\tilde{\mathcal{F}}$ be an $\mathbf{S E}(2)$-invariant vector field on a domain $W$. Then $\tilde{\mathcal{F}}$ may be lifted in a standard way to an $\mathbf{S E}(2)$-invariant vector field $\mathcal{F}$ on $\mathbf{S E}(2) \times V$ by the covering map П. More precisely, let $\Pi_{*}$ be the tangent map corresponding to $\Pi$. Then $\mathcal{F}=\Pi_{*}{ }^{-1} \tilde{\mathcal{F}}$ is the $\mathbf{S E}(2)$-invariant vector field on $\mathbf{S E}(2) \times V$. Due to (4.7), vector field $\mathcal{F}$ is invariant under the action $S(\tilde{g})$ of $\mathbf{S E}(2)$ on $\mathbf{S E}(2) \times V$. Since the group $\mathbf{S E}(2)$ acts by left shifts on the first component and identically on the second component of $\mathbf{S E}(2) \times V$, vector field $\mathcal{F}$ on $\mathbf{S E}(2) \times V$ may be represented in the form (4.2).

\section{Remarks.}

- In fact, the vector field $\mathcal{F}$ on $\mathbf{S E}(2) \times V$ is invariant under the extended symmetry group $\mathbf{S E}(2) \times \tilde{K}$. The invariance of $\mathcal{F}$ under the structural group $\{e\} \times \tilde{K}$ (see Remark following Lemma 4.3.2) just means that the vector fileds on different sheets of the covering space $\mathbf{S E}(2) \times V$, permuted by elements of the structural group $\tilde{K}$, are identical. 
- The original $n$-armed spiral wave with the period $\tau(\varepsilon)=2 \pi /\left(n \omega_{1}(\varepsilon)\right)$ corresponds to an $n \tau(\varepsilon)$-periodic orbit $\left(\varphi(t ; \varepsilon), R(t ; \varepsilon), v_{0}(\varepsilon)\right)$ of the system $(4.2)$, where $v_{0}(\varepsilon)$ is an appropriate equilibrium in the subsystem $(4.2(c))$ and $\omega\left(v_{0}(\varepsilon) ; \varepsilon\right)=\omega_{1}(\varepsilon)$. The $n \tau(\varepsilon)$-periodic orbit has a triple unit multiplier. Recall that the original spiral wave has the trivial multipliers 1 and $\mathrm{e}^{ \pm 2 \pi i / n}$ due to the symmetry, see Sect. 3.2. The case is analogous to the case of resonance $1: n$ treated in the general bifurcation theory, where an $n$-sheeted covering of the original periodic orbit having multipliers $\mathrm{e}^{ \pm 2 \pi i / n}$ is used, see Chapt. 4, $\S 21$ in [Arnold 1983] and Chapt. I, §3.2 in [Afraimovich, Arnold, Il'yashenko \& Shilnikov 1994].

\subsubsection{Symmetry of the system on $\mathrm{SE}(2) \times V$}

Let, be the generator of the symmetry group $K$ of the given $n$-armed spiral, corresponding to the rotation of the plane through $\varphi_{n}=2 \pi / n$ as defined in Sect. 3.1. Consider the restriction $\gamma=,\left.\right|_{E_{0}}$ of, to the critical eigenspace $E_{0}, E_{0}=E_{0}$.

Lemma 4.3.3 The symmetry of system (4.2) is given by the following commutation relationships

$$
\left\{\begin{array}{l}
(a) \quad \omega(\gamma v ; \epsilon)=\omega(v ; \epsilon), \\
(b) \quad a(\gamma v ; \epsilon)=\mathrm{e}^{2 \pi i / n} a(v ; \epsilon), \\
(c) \quad b(\gamma v ; \epsilon)=\gamma b(v ; \epsilon) .
\end{array}\right.
$$

Proof. The relationship (4.8(c)) follows from the $K$-invariance of the vector field reduced to $V$. Let $\mathcal{F}(v ; \epsilon)=(\omega(v ; \epsilon), a(v ; \epsilon), b(v ; \epsilon))$ be taken at point $(e, v) \in\{e\} \times V$. The linear transformation, sends $V$ onto itself and, hence, $\mathcal{F}(v ; \epsilon)$ maps to $\mathcal{F}(\gamma v ; \epsilon)$ at $(e, \gamma v) \in\{e\} \times V, \mathcal{F}(\gamma v ; \epsilon)=(\omega(\gamma v ; \epsilon), a(\gamma v ; \epsilon), b(\gamma v ; \epsilon))$. At the same time, corresponds to the left shift $g_{n}=\left(\varphi_{n}, 0\right)$ on the group $\mathbf{S E}(2)$ that rotates the $\mathbf{S E}(2)$ component $(\omega(v ; \epsilon), a(v ; \epsilon))$ through angle $\varphi_{n}$. Using $(4.5)$ with $\varphi=\varphi_{n}$, we obtain

$$
\mathrm{d} g_{n}:(\omega(v ; \epsilon), a(v ; \epsilon)) \rightarrow\left(\omega(v ; \epsilon), \mathrm{e}^{i \varphi_{n}} a(v ; \epsilon)\right)
$$

This results in $(4.8(\mathrm{a}, \mathrm{b}))$. The proof follows.

\subsection{Bifurcation group}

We call the group $G_{\text {bif }}$, generated by $\gamma$, a bifurcation group. The restriction of the group $K$ on the critical eigenspace $E_{0}$ is the homomorphism $\psi: K \rightarrow G_{\text {bif }}$ with Ker $\psi=J, J \subseteq K$, and $\psi()=,\gamma$. Thus, the bifurcation group $G_{\text {bif }}$ is isomorphic 
to the quotient group $K / J$, where $J$ acts identically on $E_{0}$ : the subgroup $J$ is the maximal spatial symmetry group of all critical eigenmodes. Let $J$ be isomorphic to $\mathbf{Z}_{d}$ ( $K$ is isomorphic to $\mathbf{Z}_{n}$ ) then the group $G_{\text {bif }}$ is isomorphic to $\mathbf{Z}_{n_{0}}$ with $n_{0}=n / d$.

\subsection{Typical critical eigenvalues}

It is known that for generic cases with commutative symmetry group, the critical eigenspace $E_{0}$ is irreducible [Golubitsky, Stewart \& Schaeffer 1988, Werner 1990]. In such cases $\operatorname{dim} E_{0}=1$ or $\operatorname{dim} E_{0}=2$. This allows one to list all generic one-parameter bifurcations of equilibria in the subsystem $(4.2(c))$ as follows.

1. $G_{\text {bif }}$ is trivial when $K$ acts identically on $E_{0}$;

2. $G_{\text {bif }}$ is isomorphic to $\mathbf{Z}_{2}$ when the restriction of $K$ on $E_{0}$ is the central symmetry ( $n$ is even);

3. $G_{\text {bif }}$ is isomorphic to $\mathbf{Z}_{n_{0}}$ when the restriction of $K$ on $E_{0}$ is generated by rotation $\gamma$ through angle $2 \pi m / n \neq \pi$. Here $n_{0}=n / d, d=\operatorname{gcd}(n, m)$ is the greatest common divisor of $m$ and $n$ and the integer $m \in\{1, \ldots, n-1\}$ is defined by the homomorphism $\psi: K \rightarrow G_{\text {bif }}\left(\right.$ or $\left.\tilde{\psi}: \mathbf{Z}_{n} \rightarrow \mathbf{Z}_{n_{0}}\right){ }^{1}$

Correspondingly to cases 1-3 the complexification of the linearisation $M=\partial_{v} b(v(0) ; 0)$ may have generically only the following eigenvalues $\lambda$ [Golubitsky et al. 1988, Werner 1990]:

I. $G_{\text {bif }}$ is trivial or the center symmetry: in either case $M$ may have the simple zero eigenvalue $\lambda=0$ or a simple complex pair $\lambda_{1,2}= \pm i \omega_{2}$;

II. $G_{\text {bif }}$ is isomorphic to $\mathbf{Z}_{n_{0}}$ with $n_{0}>2: M$ may only have a simple complex pair $\lambda_{1,2}= \pm i \omega_{2}$.

\subsection{Features of the subsystem $(4.2(\mathrm{~b}))$}

Let $G_{\text {bif }}$ be isomorphic to $\mathbf{Z}_{n_{0}}$ with $n_{0}=n / d$, see above. It follows from $(4.8(b))$ that $a\left(\gamma^{n_{0}} v ; \varepsilon\right)=a(v ; \varepsilon)=\mathrm{e}^{2 \pi i / d} a(v ; \varepsilon)$, where $\gamma^{n_{0}}=e$ is the identical transformation. If $d \neq 1$ then $a(v ; \varepsilon) \equiv 0$ and, hence, the equation $(4.2(b))$ reads, see also [Golubitsky et al. 1997, Ashwin \& Melbourne 1997],

$$
\dot{R}=0 \text {. }
$$

\footnotetext{
${ }^{1}$ In this particular case $\psi$ is also a character of the group $K$.
} 
Let $d=1$, i.e. $G_{\mathrm{bif}}$ is isomorphic to $K$ and $\gamma$ is rotation through $2 \pi m / n$ with an appropriate $m$ such that $m$ and $n$ are coprime. Using $(4.8(b))$ with $\gamma=\mathrm{e}^{2 \pi i m / n}$, we obtain $a\left(\mathrm{e}^{2 \pi i m / n} z ; \varepsilon\right)=\mathrm{e}^{2 \pi i / n} a(z ; \varepsilon)$, where $z=v_{1}+i v_{2}$ and $v=\left(v_{1}, v_{2}\right)$ is a coordinate on $E_{0}$ (recall that here $\operatorname{dim} E_{0}=2$ ). [More precisely, using the complex coordinate we get $\tilde{a}(z ; \varepsilon)=a(v(z) ; \varepsilon)$. For shortness we have omitted the tilde in the notation and will do that in what follows.] It follows that the expansion of $a(z ; \varepsilon)$ into the Taylor series in $z$ and $\bar{z}$ can have only non-zero terms $a_{k l}(\varepsilon) z^{k} \bar{z}^{l}$ with integers $k \geq 0$ and $l \geq 0$ that satisfy the Diophantine equation

$$
n s+m(k-l)=1, \quad s \in \mathbf{Z} .
$$

Recall that equation (4.10) has a countable set of integer solutions $(s, k-l)$ if and only if $m$ and $n$ are coprime, otherwise it has no solution.

\subsection{On bifurcations in the system (4.2)}

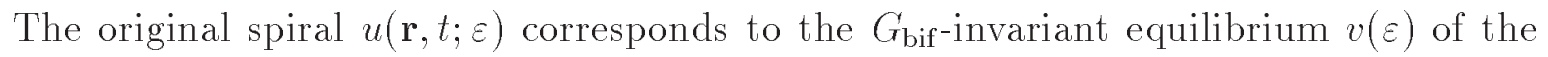
subsystem $(4.2(c)), \gamma v(\varepsilon)=v(\varepsilon)$, and the analysis of bifurcations of $u(\mathbf{r}, t ; 0)$ is equivalent to that of the equilibrium $v(0)$. Nevertheless, because we are interested in the study of both normal dynamics 'across' and the drift phenomena 'along' the spiral wave manifold we will carry out the bifurcation analysis in terms of the full system (4.2).

Spiral waves are not invariant under reflections $I_{x}:(x, y) \rightarrow(-x, y)$ and $I_{y}$ : $(x, y) \rightarrow(x,-y)$, so the same bifurcation occurs simultaneously for two spirals mapped one to the other by a reflection. Hence, it is enough to study the bifurcation for only one of them.

\section{$5 \quad$ Bifurcations of one-armed spiral waves}

Below we use the notations $C(\varepsilon)$ for the periodic orbit of $u(\mathbf{r}, t ; \varepsilon), \tau=2 \pi / \omega_{1}$ for the period, $\mu$ for the critical multiplier and $\lambda$ for the critical Floquet exponent of $C(0)$.

Since the isotropy subgroup $K$ of one-armed spiral waves is trivial, it follows from Sect. 4.5 that one-armed spirals can only undergo fold and Hopf bifurcations in generic one-parameter families of differential equations.

\subsection{Fold bifurcation}

Proposition 5.1 Let $C(0)$ have a simple $\lambda=0$. Then the normal form of (4.2) is 


$$
\left\{\begin{array}{l}
(a) \quad \dot{\phi}=\omega_{1}+\beta_{1} \zeta+\mathcal{O}\left(\varepsilon+\zeta^{2}\right), \\
(b) \quad \dot{P}=e^{i \phi}\left[a_{0}+a_{1} \zeta+\mathcal{O}\left(\varepsilon+\zeta^{2}\right)\right], \\
(c) \quad \dot{\zeta}=\varepsilon \lambda_{1}+b_{2} \zeta^{2}+\mathcal{O}\left(\varepsilon^{2}+\zeta^{3}\right),
\end{array}\right.
$$

where $\zeta \in \mathbf{R}^{1}, \omega_{1}, \beta_{1}, \lambda_{1}$ and $b_{2}$ are real constants, and $a_{0}, a_{1}$ are complex constants, $P \in \mathbf{C}$. If $\lambda_{1} \neq 0$ and $b_{2} \neq 0$ then the fold bifurcation of $C(0)$ occurs at $\varepsilon=0$.

Proof. If the nondegeneracy conditions $b_{v v}^{\prime \prime}(0,0) \neq 0$ and $b_{\varepsilon}^{\prime}(0 ; 0) \neq 0$ hold then $(4.2(c))$ can be brought to the form $(5.1(c))$ [Kuznetsov 1995, §3.3]. Expanding $\tilde{\omega}(\zeta ; \varepsilon)=$ $\omega(v(\zeta ; \varepsilon) ; \varepsilon)$ and $\tilde{a}(\zeta ; \varepsilon)=a(v(\zeta ; \varepsilon) ; \varepsilon)$ into the Taylor series in $\zeta$ and $\varepsilon$ at $\zeta=\varepsilon=0$, one can transform $(4.2(a),(b))$ to $(5.1(a),(b))$. Finally, the complete bifurcation analysis follows from the analysis of $(5.1(c))$. Indeed, the equilibria $\zeta_{1,2}(\varepsilon)= \pm\left(-\varepsilon \lambda_{1} / b_{2}\right)^{1 / 2}+$ $\mathcal{O}\left(\varepsilon^{3 / 2}\right)$ correspond to two spiral waves of (2.1), existing for $\lambda_{1} \varepsilon / b_{2} \leq 0$. The proof follows.

\section{Remarks.}

- Because of the Jordan block formed by the double unit multiplier of $C(0)$, corresponding to the rotation and critical eigenmodes at $\varepsilon=0$, the term $\beta_{1} \zeta$ cannot be removed from $(5 \cdot 1(\mathrm{a}))$.

- Equation $(5.1(b))$ can be further simplified by change of variables $\tilde{P}=P+$ $i \mathrm{e}^{i \varphi} a_{0} / \omega_{1}$ which annihilates $a_{0}$. The same is true for all normal forms obtained in this paper. This change of variables has a visual interpretation. $P$ can be viewed as the position of the spiral tip, and the definition of the tip may vary [Biktashev et al. 1996]. Then $\tilde{P}$ corresponds to such a choice of tip that places it exactly at the center of rotation at $\varepsilon=0$.

Model system 5.1 A simple differential equation, capturing most of the essential features of the bifurcation phenomenon near a critical point, is called a model system. In the case of a fold bifurcation such a model system is

$$
\left\{\begin{array}{l}
\dot{\phi}=\omega_{1}+\beta \zeta, \\
\dot{P}=\mathrm{e}^{i \phi}(a+\zeta), \\
\dot{\zeta}=\alpha-\zeta^{2}
\end{array}\right.
$$

where $\alpha$ is the bifurcation parameter and the other parameters $a, \beta$ and $\omega_{1}$ are fixed. 


\subsection{Hopf bifurcation (generic non-resonant case)}

Proposition 5.2 Let $C(0)$ have a pair of $\lambda_{1,2}= \pm i \omega_{2}$. If the non-resonance condition

$$
\omega_{1} \neq q \omega_{2}
$$

holds for all $q \in \mathbf{N}$, where $\omega_{j}>0, j=1,2$, then

1. The normal form of $(4.2)$ is

$$
\left\{\begin{array}{l}
(a) \quad \dot{\phi}=\omega(z ; \varepsilon)=\omega_{1}+\beta_{11}|z|^{2}+\mathcal{O}\left(\varepsilon+|z|^{4}\right) \\
\text { (b) } \dot{P}=\mathrm{e}^{i \phi} a(z ; \varepsilon)=\mathrm{e}^{i \phi}\left[a_{0}+a_{10} z+\mathcal{O}\left(\varepsilon+|z|^{2}\right)\right] \\
(c) \quad \dot{z}=\left(\varepsilon \lambda_{1}+i\left(\omega_{2}+\varepsilon \omega_{21}\right)\right) z+B z|z|^{2}+\mathcal{O}\left(\varepsilon^{2}|z|+|z|^{4}\right)
\end{array}\right.
$$

where $z \in \mathbf{C}, \beta_{11}, \lambda_{1}, \omega_{1}, \omega_{2}$ and $\omega_{21}$ are real constants and $a_{0}, a_{10}, B$ are complex constants.

2. If $\lambda_{1} \neq 0$ and $\operatorname{Re} B \neq 0$ then for small $\varepsilon$ a unique limit cycle of $(5.3(c))$ appears in the small neighbourhood of $z=0$.

3. Any solution $P(t ; \varepsilon)$ to $(5.3(b))$, corresponding to the cycle of $(5.3(c))$, traces out a two-periodic trajectory in a bounded domain of the complex plane.

\section{Proof.}

1) Equation $(4.2(c))$ can be brought to the form $(5.3(c))$ as in [Kuznetsov 1995, §3.5]. Making the Poincaré change of coordinates [Arnold 1983, §22],

$$
\varphi=\phi+h_{k l} z^{k} \bar{z}^{l}
$$

and using the normalised equation $\left(5.3(c)\right.$ ), one can eliminate a term $\beta_{k l} z^{k} \bar{z}^{l}$ from the expansion of $\omega(z ; \varepsilon)$ into the Taylor series in $z, \bar{z}$ and $\varepsilon$ near $z=0$ and $\varepsilon=0$ by putting

$$
h_{k l}=\frac{\beta_{k l}}{i(k-l) \omega_{2}}
$$

where $k-l \neq 0$. Keeping the minimal values $k=l=1$ when $k-l \neq 0$ does not hold, one brings $(4.2(a))$ to $(5.3(a))$. Similarly, making the change of coordinates

$$
R=P+\mathrm{e}^{i \phi} H_{k l} z^{k} \bar{z}^{l}
$$

and using both $(5.3(a))$ and $(5.3(c))$, one can eliminate a term $a_{k l} z^{k} \bar{z}^{l}$ from the expansion of $a(z ; \varepsilon)$ into the Taylor series in $z, \bar{z}$ and $\varepsilon$ near $z=0$ and $\varepsilon=0$ by putting

$$
H_{k l}=\frac{a_{k l}}{i\left(\omega_{1}+(k-l) \omega_{2}\right)},
$$

where $\omega_{1}+(k-l) \omega_{2} \neq 0$ for all $k-l \in \mathbf{Z}$ due to (5.2). Thus, due to the non-resonance condition (5.2) all terms $a_{k l} z^{k} \bar{z}^{l}$ are non-resonant in the sense of [Arnold 1983, §22]. 
Keeping the term corresponding to $k=1$ and $l=0$ and, in turn, removing the term with $k=0$ and $l=1$, we get $(5.3(b))$.

2) Equation $(5.3(c))$ has a unique periodic orbit near $z=0$ for small $\varepsilon>0$ or $\varepsilon<0$ [Kuznetsov 1995, §3.7]. Denote by $z(t ; \varepsilon)$ the corresponding periodic solution and by $\tau_{2}(\varepsilon)$ its period.

3) Let us represent $\omega(t ; \varepsilon)=\omega(z(t ; \varepsilon) ; \varepsilon)$ in the form $\omega(t ; \varepsilon)=\hat{\omega}_{1}(\varepsilon)+\tilde{\omega}_{1}(t ; \varepsilon)$, where

$$
\hat{\omega}_{1}(\varepsilon)=\omega_{1}+\mathcal{O}(\varepsilon)
$$

is the mean value of $\omega(t ; \varepsilon)$ and $\tilde{\omega}_{1}(t ; \varepsilon)=\mathcal{O}(\varepsilon)$ is $\tau_{2}(\varepsilon)$-periodic. Integrating $(5.3(a))$ and then substituting $\phi(t ; \varepsilon)=\phi_{0}+\hat{\omega}_{1}(\varepsilon) t+\Omega(t ; \varepsilon)$ into $(5.3(b))$, one gets

$$
\left\{\begin{array}{l}
\dot{P}=A(t ; \varepsilon) \mathrm{e}^{i \hat{\omega}_{1}(\varepsilon) t}, \\
\quad A(t ; \varepsilon)=a(z(t ; \varepsilon) ; \varepsilon) \mathrm{e}^{i\left(\phi_{0}+\Omega(t ; \varepsilon)\right)}, \quad \Omega(t ; \varepsilon)=\int_{0}^{t} \tilde{\omega}_{1}(s ; \varepsilon) \mathrm{d} s,
\end{array}\right.
$$

where both $A(t ; \varepsilon)$ and $\Omega(t ; \varepsilon)$ are $\tau_{2}(\varepsilon)$-periodic. Expanding $A(t ; \varepsilon)$ into the uniformly convergent Fourier series,

$$
A(t ; \varepsilon)=\sum_{-\infty}^{\infty} A_{k}(\varepsilon) \mathrm{e}^{i k \omega_{2}(\varepsilon) t}
$$

where $\omega_{2}(\varepsilon)=2 \pi / \tau_{2}(\varepsilon), \omega_{2}(0)=\omega_{2}$, and then integrating (5.9), one obtains

$$
P(t ; \varepsilon)=P_{0}+\sum_{-\infty}^{\infty} \frac{A_{k}(\varepsilon) \mathrm{e}^{i\left(\hat{\omega}_{1}(\varepsilon)+k \omega_{2}(\varepsilon)\right) t}}{i\left(\hat{\omega}_{1}(\varepsilon)+k \omega_{2}(\varepsilon)\right)},
$$

Due to (5.2) and (5.8), there exists a constant $c$ such that for sufficiently small $\varepsilon$ and any $k \in \mathbf{Z}$, the inequality $\left|\hat{\omega}_{1}(\varepsilon)+k \omega_{2}(\varepsilon)\right| \geq c$ holds. Since series (5.10) uniformly converges, series (5.11) also uniformly converges. Thus, $P(t ; \varepsilon)$ is a continuous biperiodic and, therefore, bounded complex-valued function. The proof follows.

Remark. Biperiodic solutions on $\mathcal{W}_{c}(\varepsilon)$ correspond to modulated (meandering) spiral waves $u\left(\mathbf{r}, \hat{\omega}_{1}(\varepsilon) t, \omega_{2}(\varepsilon) t ; \varepsilon\right)$ in the original system $(2.1)$.

Model system 5.2 A model system for a non-resonant Hopf bifurcation is

$$
\left\{\begin{array}{l}
\dot{\phi}=\omega_{1} \\
\dot{P}=\mathrm{e}^{i \phi}(a+z) \\
\dot{z}=\left(\alpha+i \omega_{2}\right) z-z|z|^{2}
\end{array}\right.
$$

where $\alpha$ is the bifurcation parameter and the parameters $a, \omega_{1}$ and $\omega_{2}$ are fixed. 


\subsection{Hopf bifurcation (non-generic resonant case)}

Let a complex-conjugate pair of multipliers $\mu_{1,2}(\varepsilon)$ of a periodic orbit $C(\varepsilon)$ cross the unit circle in the complex plane at 'rational' points $\mu_{1,2}=\mathrm{e}^{ \pm i \omega_{2} \tau}=\mathrm{e}^{ \pm 2 \pi i / q}$ at $\varepsilon=0$, that is a $1: q$-resonance holds. Here $\tau=2 \pi / \omega_{1}$ and $\omega_{1}=q \omega_{2}$. As in the general theory [Afraimovich et al. 1994, Arnold 1983, Guckenheimer \& Holmes 1986, Kuznetsov 1995] to carry out the complete bifurcation analysis of the resonant case we need to involve a second bifurcation parameter. Let $\varepsilon=\left(\varepsilon_{1}, \varepsilon_{2}\right)$.

Proposition 5.3 Let $C(0)$ have a pair of $\lambda_{1,2}= \pm i \omega_{2}$ such that for some $q \in \mathbf{N}$

$$
\omega_{1}=q \omega_{2}
$$

holds, where $\omega_{j}>0, j=1,2$, then

1. The normal form of $(4.2)$ is

$$
\left\{\begin{array}{l}
(a) \quad \dot{\phi}=\omega(z ; \varepsilon)=\omega_{1}(\varepsilon)+\beta(\varepsilon)|z|^{2}+\mathcal{O}\left(|z|^{4}\right), \\
(b) \quad \dot{P}=\mathrm{e}^{i \phi} a(z ; \varepsilon)=\mathrm{e}^{i \phi}\left[a_{0}(\varepsilon)+a_{q}(\varepsilon) \bar{z}^{q}+\mathcal{O}\left(|z|^{q+2}\right)\right] \\
(c) \quad \dot{z}=\left(\lambda(\varepsilon)+i \omega_{2}(\varepsilon)\right) z+B(\varepsilon) z|z|^{2}+\mathcal{O}\left(|z|^{4}\right),
\end{array}\right.
$$

where $z \in \mathbf{C}, \beta(\varepsilon), \lambda(\varepsilon), \omega_{1}(\varepsilon), \omega_{2}(\varepsilon)$ are real and $a_{0}(\varepsilon), a_{q}(\varepsilon), B(\varepsilon)$ are complex.

2. If $\lambda_{\varepsilon_{1}}^{\prime}(0)^{2}+\lambda_{\varepsilon_{2}}^{\prime}(0)^{2} \neq 0$ and $\operatorname{Re} B(0) \neq 0$ then a unique limit cycle $z(t ; \varepsilon)$ of $(5.13(c))$ with the period $\tau(\varepsilon)=2 \pi / \omega_{2}(\varepsilon)$ appears near $z=0$, where $\omega_{2}(0)=\omega_{2}$.

3. Let $\hat{\omega}_{1}(\varepsilon)$ be the mean value of $\omega(t ; \varepsilon)=\omega(z(t ; \varepsilon) ; \varepsilon)$, where $\hat{\omega}_{1}(0)=\omega_{1}$, and let $\mathcal{L}$ be the resonance locus in the small neighbourhood of $\varepsilon=0$ in the plane $\left(\varepsilon_{1}, \varepsilon_{2}\right)$,

$$
\mathcal{L}=\left\{\left(\varepsilon_{1}, \varepsilon_{2}\right) \mid \hat{\omega}_{1}\left(\varepsilon_{1}, \varepsilon_{2}\right)-q \omega_{2}\left(\varepsilon_{1}, \varepsilon_{2}\right)=0\right\}
$$

Any solution $P(t ; \varepsilon)$ to $(5.13(b))$, corresponding to $z(t ; \varepsilon)$, is bounded for all small $\varepsilon \notin \mathcal{L}$. If $\left(\varepsilon_{1}, \varepsilon_{2}\right) \in \mathcal{L}$ then $P(t ; \varepsilon)$ undergoes a secular growth as $P(t ; \varepsilon)=P_{0}+c_{1} t+\tilde{P}(t ; \varepsilon)$, where $|\tilde{P}(t ; \varepsilon)|$ is bounded.

Proof. The proof is similar to the non-resonant case. One can use the Poincaré change of variables like (5.4) and (5.6), which now depend on $\varepsilon$, to eliminate non-resonant nonlinear terms from the Taylor series in $z$ and $\bar{z}$ of $\omega(z ; \varepsilon)$ and $a(z ; \varepsilon)$ near $z=0$, respectively. For these changes of variables to be smooth in $\varepsilon$ at $\varepsilon=0$, the values of $k$ and $l$ must satisfy the conditions $k-l \neq 0$ for $(5.5)$ and $\omega_{1}+(k-l) \omega_{2} \neq 0$ for $(5.7)$ at $\varepsilon=0$. Keeping minimal $k=0$ and $l=q$, when the condition $\omega_{1}+(k-l) \omega_{2} \neq 0$ 
does not hold at $\varepsilon=0$, and using $(5.13(a))$ and $(5.13(c))$, one can transform $(4.2(b))$ to $(5.13(b))$. The statements on the behaviour of $P(t ; \varepsilon)$ when $\varepsilon \notin \mathcal{L}$ or $\varepsilon \in \mathcal{L}$ follow from the direct integration of $(5.13(b))$.

Remark. The complex-valued function $P(t)=P_{0}+c_{1} t+\tilde{P}(t ; \varepsilon)$ can be interpreted as describing the resonant drift of the wavetip across the plane. Let $P(t)=P_{1}(t)+i P_{2}(t)$ then $\left(P_{1}(t), P_{2}(t)\right)$ can be viewed as coordinates of the wavetip [Biktashev et al. 1996]. In some applications such drifting spiral waves are called modulated travelling waves [Barkley 1995].

Model system 5.3 The following $(\alpha-\beta)$-model system

$$
\left\{\begin{array}{l}
\dot{\phi}=\omega_{1}+\beta \\
\dot{P}=\mathrm{e}^{i \phi}\left(a+\bar{z}^{q}\right), \\
\dot{z}=\left(\alpha+i \omega_{2}\right) z-z|z|^{2}
\end{array}\right.
$$

captures all essential features of the resonant drift phenomenon near a resonance of order $q \in \mathbf{N}, \omega_{1}=q \omega_{2}$. In this case both periodic solution $z(t ; \alpha)=\alpha^{1 / 2} \mathrm{e}^{i \omega_{2} t}$ and resonance locus $\mathcal{L}=\{\beta=0\}$ in the $(\alpha-\beta)$-parameter plane take the simplest forms.

\section{Bifurcations of multi-armed spiral waves}

Throughout this section $u(\mathbf{r}, t ; \varepsilon)$ is assumed to be an $n$-armed spiral wave with the spatial symmetry $K$ isomorphic to $\mathbf{Z}_{n}, n \geq 2$.

\subsection{Fold bifurcation}

Proposition 6.1 Let $C(0)$ have a simple $\lambda=0$ and let the bifurcation group $G_{\mathrm{bif}}$ be trivial. Then the fold bifurcation takes place and the normal form of (4.2) is

$$
\left\{\begin{array}{l}
(a) \quad \dot{\phi}=\omega_{1}+\beta_{1} \zeta+\mathcal{O}\left(\varepsilon+\zeta^{2}\right) \\
\text { (b) } \dot{P}=0 \\
\text { (c) } \dot{\zeta}=\varepsilon \lambda_{1}+b_{2} \zeta^{2}+\mathcal{O}\left(\varepsilon^{2}+\zeta^{3}\right)
\end{array}\right.
$$

where $\zeta \in \mathbf{R}^{1}, \omega_{1}, \beta_{1}, \lambda_{1} \neq 0$ and $b_{2} \neq 0$ are real constants.

The proof of Proposition 6.1 is similar to that of Proposition 5.1 with the only difference that $(5.1(b))$ is replaced by $(6.15(b))$ due to $(4.9)$ since $G_{\text {bif }}$ is not isomorphic to $K$. 
Model system 6.1 In the case of multi-armed spiral waves a fold model system is

$$
\left\{\begin{array}{l}
\dot{\phi}=\omega_{1}+\beta \zeta \\
\dot{P}=0 \\
\dot{\zeta}=\alpha-\zeta^{2}
\end{array}\right.
$$

\subsection{Pitchfork and period doubling bifurcation}

Proposition 6.2 Let $C(0)$ with the period $\tau=2 \pi /\left(n \omega_{1}\right)$ have a simple $\lambda=0$ and let $G_{\mathrm{bif}}$ be the central symmetry. Then

1. The normal form of $(4.2)$ is

$$
\left\{\begin{array}{l}
(a) \quad \dot{\phi}=\omega(\zeta ; \varepsilon)=\omega_{1}+\beta_{2} \zeta^{2}+\mathcal{O}\left(\varepsilon+\zeta^{4}\right), \\
(b) \quad \dot{P}=\mathrm{e}^{i \phi} a(\zeta ; \varepsilon)=\mathrm{e}^{i \phi}\left[a_{1} \zeta+\mathcal{O}\left(\varepsilon \zeta+\zeta^{3}\right)\right] \delta(n-2), \\
\text { (c) } \dot{\zeta}=\varepsilon \lambda_{1} \zeta+b_{3} \zeta^{3}+\mathcal{O}\left(\varepsilon^{2} \zeta+\zeta^{5}\right)
\end{array}\right.
$$

where $\zeta \in \mathbf{R}^{1}, \omega_{1}, \beta_{2}, \lambda_{1}$ and $b_{3}$ are real constants and $a_{1}$ is a complex constant. Here $\delta(n-2)=1$ if $n=2$ and 0 otherwise.

2. If $\lambda_{1} \neq 0$ and $b_{3} \neq 0$ then a pitchfork bifurcation occurs, leading to a pair of congruent $\mathbf{Z}_{n / 2}$-symmetric $n$-armed spiral waves with the period $\tau_{1,2}(\varepsilon) \rightarrow 2 \tau(0)$ as $\varepsilon \rightarrow 0$.

Proof. Equation (4.2(c)) can be brought to the form (6.16(c)) [Kuznetsov 1995, §7.4.3]. If $n=2$ then $\omega(-\zeta ; \varepsilon) \equiv \omega(\zeta ; \varepsilon)$ and $a(-\zeta ; \varepsilon) \equiv-a(\zeta ; \varepsilon)$ due to $(4.8)$, otherwise $a(\zeta ; \varepsilon) \equiv 0$. The two equilibria $\zeta_{1,2}(\varepsilon)$ of $(6.16(\mathrm{c}))$ correspond to two periodic orbits $C_{1,2}(\varepsilon)$ in (2.1). Since $C_{2}(\varepsilon) \neq C_{1}(\varepsilon)$ it follows that $C_{2}(\varepsilon)=, C_{1}(\varepsilon)$. These periodic orbits correspond to two $\mathbf{Z}_{d}$-symmetric spiral waves, see Sect. 3.1, which have the periods $\tau_{1,2}(\varepsilon)=2 \pi / d \omega_{1,2}(\varepsilon) \rightarrow 2 \pi / d \omega_{1}=2 \tau(0)$ as $\varepsilon \rightarrow 0$, where $d=n / 2$. The proof follows.

Remark. The spatial symmetry $\tilde{K}$ of the new bifurcating spiral waves is the inverse image of the unit $e \in G_{\text {bif }}$ under homomorphism $\psi: K \rightarrow G_{\text {bif }}, \tilde{K}^{\prime}=\psi^{-1}(e)$.

Model system 6.2 For a pitchfork (period doubling) bifurcation a model system is

$$
\left\{\begin{array}{l}
\dot{\phi}=\omega_{1}+\beta \zeta^{2} \\
\dot{P}=\mathrm{e}^{i \phi} \zeta \delta(n-2) \\
\dot{\zeta}=\alpha \zeta-\zeta^{3}
\end{array}\right.
$$




\subsection{Hopf bifurcation (generic non-resonant case)}

We consider the case where the bifurcation group $G_{\text {bif }}$ is isomorphic to the full spatial symmetry group $K$ of the original $n$-armed spiral. The case where $G_{\text {bif }}$ is isomorphic to a subgroup of $K$ is analogous and is not of much interest.

Proposition 6.3 Let $C(0)$ have a pair of $\lambda_{1,2}= \pm i \omega_{2}$ and let $\gamma=\psi()=,\mathrm{e}^{2 \pi i m / n}$, where $\psi: K \rightarrow G_{\mathrm{bif}}$ is an isomorphism.

1. If

$$
\omega_{1} \neq q \omega_{2}
$$

for all $q \in \mathbf{N}$ satisfying the Diophantine equation

$$
n s-m q=1, \quad s \in \mathbf{Z},
$$

then a normal form of (4.2) is

$$
\left\{\begin{array}{l}
(a) \quad \dot{\phi}=\omega(z ; \varepsilon)=\omega_{1}+\beta_{11}|z|^{2}+\mathcal{O}\left(\varepsilon+|z|^{4}\right) \\
\text { (b) } \dot{P}=\mathrm{e}^{i \phi} a(z ; \varepsilon)=\mathrm{e}^{i \phi}\left[a_{k_{0}} z^{k_{0}}+\mathcal{O}\left(\varepsilon|z|^{k_{0}}+|z|^{M}\right)\right] \\
(c) \quad \dot{z}=\left(\varepsilon \lambda_{1}+i\left(\omega_{2}+\varepsilon \omega_{21}\right)\right) z+B z|z|^{2}+\mathcal{O}\left(\varepsilon^{2}|z|+|z|^{4}\right) .
\end{array}\right.
$$

Here $k_{0} \geq 0$ is the minimal positive solution to (4.10) with $l=0$ and $M>k_{0}$ is the degree of the next non-vanishing term at $\varepsilon=0$. Constants $\omega_{1}, \omega_{2}, \omega_{21}, \beta_{11}, \lambda_{1}$ are real, $a_{k_{0}}, B$ are complex and $z \in \mathbf{C}$.

2. If $\lambda_{1} \neq 0$ and $\operatorname{Re} B \neq 0$ then a unique limit cycle of $(6.19(c))$ appears near $z=0$.

3. Any solution $P(t ; \varepsilon)$ to $(6.19(b))$, corresponding to the unique cycle of $(6.19(c))$ near $z=0$, traces out a two-periodic bounded trajectory in the complex plane.

Proof. The proposition can be proved in the same way as Proposition 5.2. Recall that all non-vanishing terms $a_{k, l}(\varepsilon) z^{k} \bar{z}^{l}$ in the Taylor series of $a(z ; \varepsilon)$ correspond to integers $k$ and $l$ that satisfy the equation (6.18) with $q=l-k$, see (4.10). Due to (6.17) all such terms are non-resonant and, hence, can be eliminated by using the Poincaré change of variables like (5.6). Keeping explicitly only terms with the smallest degrees, one can obtain (6.19).

Let $z(t ; \varepsilon)$ be a periodic solution to $(6.19(\mathrm{c}))$ with the period $\tau_{2}(\varepsilon)=2 \pi / \omega_{2}(\varepsilon)$, corresponding to the periodic orbit emanating from $z=0$ at $\varepsilon=0$. Since this periodic orbit is unique, the following identity holds 


$$
\gamma z(t ; \varepsilon) \equiv z\left(t+\frac{2 \pi m}{n \omega_{2}(\varepsilon)} ; \varepsilon\right)
$$

Let $P(t ; \varepsilon)$ be a solution to the equation $(6.19(\mathrm{~b}))$ that can be rewritten in the equivalent form (5.9). Then due to $(4.8(a),(b))$ and $(6.20)$ the complex-valued function $A(t ; \varepsilon)$, see (5.9) and (5.10), satisfies

$$
A\left(t+\frac{2 \pi m}{n \omega_{2}(\varepsilon)} ; \varepsilon\right)=\mathrm{e}^{2 \pi i / n} A(t ; \varepsilon) .
$$

It follows from (6.21) that Fourier coefficients $A_{k}(\varepsilon)$, see (5.10), are non-zero for all $k \in \mathbf{Z}$ that satisfy $n s-m k=1, s \in \mathbf{Z}$, otherwise $A_{k}(\varepsilon) \equiv 0$. It follows from (6.18) that for all such $k$ one has $\left|\hat{\omega}_{1}(\varepsilon)-k \omega_{2}(\varepsilon)\right| \geq c$, that is $P(t ; \varepsilon)$, see $(5.11)$, is bounded. Here $\hat{\omega}_{1}(\varepsilon)$ is the mean value of $\omega(z(t ; \varepsilon) ; \varepsilon)$. The proof follows.

Model system 6.3 A model system for a non-resonant Hopf bifurcation is

$$
\left\{\begin{array}{l}
\dot{\phi}=\omega_{1} \\
\dot{P}=\mathrm{e}^{i \phi} z^{k} \delta\left(n-n_{0}\right) \\
\dot{z}=\left(\alpha+i \omega_{2}\right) z-z|z|^{2}
\end{array}\right.
$$

Here $n$ is the order of the spatial symmetry $K$ and $n_{0}$ is the order of the bifurcation group $G_{\mathrm{bif}} ; \delta\left(n-n_{0}\right)=1$ if $n_{0}=n$ and $\delta\left(n-n_{0}\right)=0$ if $n_{0}<n ; k$ is the smallest solution to (4.10) with $l=0$ for the given $m$.

\subsection{Hopf bifurcation (non-generic resonant case)}

Proposition 6.4 Let $C(0)$ have a pair of $\lambda_{1,2}= \pm i \omega_{2}$ and let $\gamma=\mathrm{e}^{2 \pi i m / n}$. If $\omega_{1}=q \omega_{2}$ for some $q \in \mathbf{N}$ satisfying (6.18) then

1. The normal form of $(4.2)$ is

$$
\left\{\begin{array}{lll}
(a) & \dot{\phi}=\omega_{1}(\varepsilon)+\beta_{11}(\varepsilon)|z|^{2}+\mathcal{O}\left(|z|^{4}\right), & \varepsilon=\left(\varepsilon_{1}, \varepsilon_{2}\right), \\
(b) & \dot{P}=\mathrm{e}^{i \phi}\left[a_{q}(\varepsilon) \bar{z}^{q}+\mathcal{O}\left(|z|^{M}\right)\right], & M>q, \\
(c) & \dot{z}=\left(\lambda(\varepsilon)+i \omega_{2}(\varepsilon)\right) z+B(\varepsilon) z|z|^{2}+\mathcal{O}\left(|z|^{4}\right) .
\end{array}\right.
$$

Here $z \in \mathbf{C}, \omega_{1}(\varepsilon), \omega_{2}(\varepsilon), \beta_{11}(\varepsilon)$ and $\lambda(\varepsilon)$ are real, $a_{q}(\varepsilon)$ and $B(\varepsilon)$ are complex.

2. If $\lambda_{\varepsilon_{1}}^{\prime}(0)^{2}+\lambda_{\varepsilon_{2}}^{\prime}(0)^{2} \neq 0$ and $\operatorname{Re} B(0) \neq 0$ then a unique limit cycle $z(t ; \varepsilon)$ of $(6.22(c))$ with the period $\tau(\varepsilon)=2 \pi / \omega_{2}(\varepsilon)$ appears near $z=0$, where $\omega_{2}(0)=\omega_{2}$. 
3. Let $\hat{\omega}_{1}(\varepsilon)$ be the mean value of $\omega(t ; \varepsilon)=\omega(z(t ; \varepsilon) ; \varepsilon)$. Any solution $P(t ; \varepsilon)$ to $(6.22(b))$, corresponding to $z(t ; \varepsilon)$, is bounded for all small $\left(\varepsilon_{1}, \varepsilon_{2}\right) \notin \mathcal{L}$ as in $(5.14)$. If $\left(\varepsilon_{1}, \varepsilon_{2}\right) \in \mathcal{L}$ then $P(t ; \varepsilon)$ undergoes a secular growth as $P(t ; \varepsilon)=P_{0}+c_{1} t+\tilde{P}(t ; \varepsilon)$, where $|\tilde{P}(t ; \varepsilon)|$ is bounded.

Proof is similar to those for Propositions 5.3 and 6.3.

Model system 6.4 A ( $\alpha$ - $\beta)$-model system for a resonant Hopf bifurcation is

$$
\left\{\begin{array}{l}
\dot{\phi}=\omega_{1}+\beta \\
\dot{P}=\mathrm{e}^{i \phi} \bar{z}^{q} \delta\left(n-n_{0}\right) \\
\dot{z}=\left(\alpha+i \omega_{2}\right) z-z|z|^{2}
\end{array}\right.
$$

\section{Discussion}

In this paper we have considered the simplest local one-parameter (fold, Hopf and pitchfork) and two-parameters (resonant Hopf) bifurcations of spiral waves uniformly rotating in the plane.

Numerical experiments have already revealed one-parameter bifurcations that are not covered by this list. Examples are the 'rotor boundary' $\partial R$ and the 'complex regimes boundary' $\partial C$, one-parameter loci of the two-parameter bifurcation diagram given by Winfree [1991].

At the rotor boundary, the rigidly rotating spiral wave disappears and this might be viewed as the fold bifurcation. However, in the known examples, approaching this boundary is accompanied by the increase of rotation period, which is not characteristic of the normal form (5.1). So, this probably should be attributed to an appropriate non-local one-parameter bifurcation.

The mechanism hypothesised in [Elkin, Biktashev \& Holden 1998] suggests that this boundary does not correspond to any bifurcation in the reduced system $(4.2(\mathrm{c}))$, but to vanishing of the value $\omega_{1}$ of corresponding equilibrium. The solution is then a propagating, rather than rotating broken plane wave. The main difficulty here is that in the norm of $B C_{\text {unif }}\left(\mathbf{R}^{2}, \mathbf{R}^{l}\right)$, rotations of the plane act discontinuously on such a solution. This singularity cannot be 'unfolded' within the functional space considered.

Another example, the 'hypermeander' boundary [Winfree 1991], should probably correspond to appropriate bifurcations of meandering spirals, i.e. bifurcations of periodic orbits in the reduced system, analogous to (4.2(c)) [Biktashev et al. 1996, Biktashev \& Holden 1998]. 
Concerning the two-parameters diagram by Winfree [1991], it is also interesting to notice that the $(s-w)$-model system suggested in [Barkley \& Keverkidis 1994] to describe this diagram coincides with one of the classical model systems studied within the general bifurcation theory. Namely the $(s-w)$-model system belongs to the Lotka-Volterra class of systems used in the analysis of the Hopf-Hopf (double Hopf) bifurcation [Afraimovich et al. 1994, Guckenheimer \& Holmes 1986, Kuznetsov 1995]. Notice that the Hopf-Hopf model system may exhibit invariant tori and chaotic motions, as well as Neimark-Sacker and Shil'nikov homoclinic bifurcations. This model system may also admit, in some difficult cases, a periodic orbit with the period that tends to infinity. Such non-local evolution of the periodic orbit cannot be completely described by any of the abovementioned bifurcations in the model system.

As mentioned in the Introduction, the centre manifold approach to the spiral wave theory has not been rigorously justified, as yet, as the structure of the spectrum of the linearised problem has not been studied in detail. There is numerical evidence that critical eigenvalues in the Hopf bifurcation are isolated [Barkley 1992]; however, the behaviour of the corresponding eigenmodes in those calculations seemed paradoxical. Namely, their real and imaginary parts seemed to belong to the functional space $B C_{\text {unif }}\left(\mathbf{R}^{2}, \mathbf{R}^{l}\right)$ only in the supercritical but not in the subcritical region. The calculations were, naturally, performed in a bounded, and a relatively small, region. This may have excluded possible complications related to the spectrum associated with long-wave modulations, which could reveal themselves only in a medium many wavelengths large.

\section{Acknowledgements}

We thank D. Barkley and M. Golubitsky for helpful discussions. This work was supported, in part, by grants from EPSRC-ANM GR/K49775, Wellcome Trust 045192 and Russian Foundation for Basic Research 96-01-00592.

\section{References}

Afraimovich, V. S., Arnold, V. I., Il'yashenko, Y. S. \& Shilnikov, L. P. [1994], Bifurcation theory, in V. I. Arnold \& et al., eds, 'Dynamical Systems-5', Encyclopaedia of Mathematical Sciences, Springer, Berlin.

Arnold, V. [1983], Geometrical Methods in the Theory of Ordinary Differential Equations, Vol. 250 of A Series of Comprehensive Studies Math., Springer, New York. 
Ashwin, P. \& Melbourne, I. [1997], 'Noncompact drift for relative equilibria and relative periodic orbits', Nonlinearity 10(3), 595-616.

Barkley, D. [1992], 'Linear stability analysis of rotating spiral waves in excitable media', Phys. Rev. Lett. 68, 2090-2093.

Barkley, D. [1995], Spiral meandering, in R. Kapral \& K. Showalter, eds, 'Chemical Waves and Patterns', Kluwer, pp. 163-190.

Barkley, D. \& Keverkidis, I. G. [1994], 'A dynamical systems approach to spiral wave dynamics', Chaos 4(3), 1-8.

Biktashev, V. N. \& Holden, A. V. [1998], 'Deterministic Brownian motion in the hypermeander of spiral waves', Physica D 116(3-4), 342-354.

Biktashev, V. N., Holden, A. V. \& Nikolaev, E. V. [1996], 'Spiral wave meander and symmetry of the plane', Int. J. of Bifurcation and Chaos 6(12), 2433-2440.

Dubrovin, B. A., Fomenko, A. T. \& Novikov, S. P. [1984], Modern geometry - methods and applications. Part 2: The Geometry and Topology of Manifolds, Springer, New York.

Elkin, Y. E., Biktashev, V. N. \& Holden, A. V. [1998], 'On the movement of excitation wave breaks', Chaos Solitons $8:$ Fractals 9(9), 1597-1610.

Fiedler, B., Sandstede, B., Scheel, A. \& Wulff, C. [1996], 'Bifurcation from relative equilibria of noncompact group actions: Skew products, meanders and drifts', Documenta Math. 1, 479-505.

Golubitsky, M., LeBlanc, V. G. \& Melbourne, I. [1997], 'Meandering of the spiral tip - an alternative approach', J. Nonlinear Sci. 133(1), 30-48.

Golubitsky, M., Stewart, I. \& Schaeffer, D. G. [1988], Singularities and Groups in Bifurcation Theory II, Vol. 69 of Appl. Math. Sci., Springer, New York.

Guckenheimer, J. \& Holmes, P. [1986], Nonlinear Oscillations, Dynamical Systems and Bifurcations of Vector Fields, Vol. 42 of Appl. Math. Sci., Springer, New York.

Henry, D. [1981], Geometric Theory of Semilinear Parabolic Equations, Vol. 840 of Lecture Notes Math., Springer, New York.

Kuznetsov, Y. A. [1995], Elements of Applied Bifurcation Theory, Springer, New York. 
Mielke, A. [1991], 'Hamiltonian and Lagrangian flows in center manifolds — with applications to elliptic variational problems', Lect. Notes in Math. 1489.

Nikolaev, E. V. \& Shnol, E. E. [1998a], 'Bifurcations of cycles in systems of differential equations with a finite symmetry group I', Journal of Dynamical and Control Systems 4, 315-341.

Nikolaev, E. V. \& Shnol, E. E. [1998b], 'Bifurcations of cycles in systems of differential equations with a finite symmetry group II', Journal of Dynamical and Control Systems 4, 343-363.

Ruelle, D. [1973], 'Bifurcations in the presence of a symmetry group', Arch. Rational Mech. Anal. 51, 136-152.

Sandstede, B., Scheel, A. \& Wulff, C. [1997], 'Dynamics of spiral waves on unbounded domains using center-manifold reductions', J. Diff. Eq. 141(1), 122-149.

Werner, B. [1990], Eigenvalue problems with the symmetry of a group and bifurcations, in D. Roose, B. De Dier \& A. Spence, eds, 'Continuation and Bifurcations: Numerical Techniques and Applications', Vol. 313 of NATO ASI Series, Series C: Mathematical and Physical Sciences, Kluwer, pp. 71-88.

Winfree, A. T. [1972], 'Spiral waves of chemical activity', Science 175, 634-636.

Winfree, A. T. [1973], 'Scroll-shaped waves of chemical activity in three dimensions', Science 181, 937-939.

Winfree, A. T. [1991], 'Varieties of spiral wave behaviour — an experimentalist's approach to the theory of excitable media', Chaos 1, 303-334.

Wulff, C. [1996], Bifurcation theory of meandering spiral waves, in Z. Parisi, S. C. Müller \& W. Zimmermann, eds, 'Nonlinear Physics of Complex Systems - Current Status and Future Trends', Springer, Berlin.

Zaikin, A. N. \& Zhabotinsky, A. M. [1970], 'Concentration wave propagation in twodimensional liquid-phase self-oscillating systems', Nature 225, 535-537.

Zykov, V. S. [1986], 'Cycloidal circulation of spiral waves in excitable medium', Biofizika 31(5), 862-865. 